\title{
تأثير الطريقة التركيبية على مهارة القراءة: \\ بحث تجريبي في الملدرسة الابتدائية الإسلامية الحكومية الواحدة تاناه داتار
}

\author{
Septika Rudiamon \\ e-mail: septikarudiamon02@gmail.com \\ Universitas Islam Negeri Imam Bonjol Padang \\ Yasmadi \\ e-mail: yasmadi@uinib.ac.id \\ Universitas Islam Negeri Imam Bonjol Padang \\ Yufni Faisol \\ e-mail: yufni_faisol@yahoo.com \\ Universitas Islam Negeri Imam Bonjol Padang
}

ملخص: الغرض من هذا البحث لمعرفة تأثير الطريقة التركيبية على مهارة القراءة لدى التلاميذ في المدرسة الابتدائية الإسلامية الحكومية الواحدة تاناه داتار. استخدم الباحث منهج شباه تجريبي مع تصميم مجموعـة ضابطة غير معادلة. وكان المجتمع OV؛ طالبًا ، بينما تكونت العينة من . إلى مجموعتين ، و T0 طالبًا في المجموعة التجريبية تم تدريسهم باستخدام طريقة تركيبية و 0 م طالبًافي المجموعـة الضابطة الذين تم تعليمههم باستخدام الطريقة السمعياة الشفهية. تم جمع البيانات عن طريق إجراء الاختبارات. بعد ذلك ، تم تحليل البيانات باستخدام اختبار to. وأظهرت النتائج أن نتيـجـة

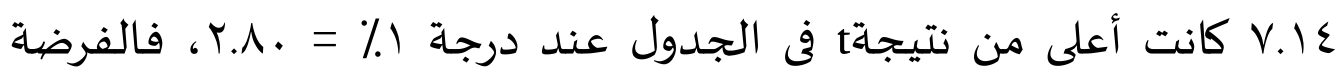
العدمية مردودة والفرضية التبادلية مقبولة. فالخلاصية أن الطريقة التركيبية تؤثر على مهارة القراءة إيجابيا في هذه المدرسـة. الكلمات الأسـاسية: الطريقة التركيبيةة، الطريقة السمعية الشفهية، مهارة القراءة، العربية، المدرسـة الابتدائية الإسلامية.

Abstract: This study aims to determine the effect of synthesis method on students' Arabic reading proficiency at MIN 1 Tanah Datar. Researchers used a quasi-experimental method with a non-equivalent control group design.The population was 457 students, while the sample consisted of 50 students divided into two groups, 25 students were in the experimental group who were taught using synthesis method and 25 other students were in the control group who were tanght using the audio-lingual method. Data were collected by giving tests. Then, the data were 
analyzed using t test. The results showed that the value of to $=7.14$ was bigher than the t table at the degree of $1 \%=2.80$, so the null hypothesis $(\mathrm{Ho})$ was rejected and the alternative bypothesis (Ha) was accepted. As conclusion, the synthesis method could positively affect students' Arabic reading proficiency in this school.

Key words: Synthesis method, audio lingual method, reading proficiency, Arabic language, Islamic primary school

ظهرت من التلاميذ الأخطاء عن قراءة

نص اللغة العربية يعني في مخارج

الحروف واللهجات، ولا يستطيع

التلاميذ أن يختلفوا صوت الحروف

وصفتها. وقامت المتحان اليومي

والامتحان النصفي للمرحلة والامتحان

الأول للمرحلة وأن تكون نتيجة تعليم

مهارة قراءتهم منخفضية، منذ الامتحان

في اليوم الأول إلى الامتحان في اليوم

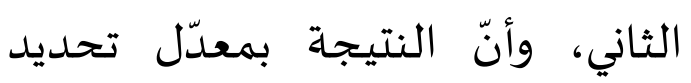

معايير الكفاءة (KKM) فى تلك المدرسـة

خمسـة وسبعون. ومن خمسين تلميذا في

تلك المدرسة وجد حول سبعة عشرة

تلميذا نتيجة فوق معدّل تحديد معايير

الكفاءة (KKM)، وثلاث وثلاثين تلميذا

وجد نتيجة تحت معدّل تحديد معايير

الكفاءة (KKM). تمّثثل هذه البيانات

$$
\text { فيما يأتي: }
$$

\begin{tabular}{|c|c|c|c|c|}
\hline \multicolumn{5}{|c|}{ الجدول الأول } \\
\hline غاجح & ناجح & مجتمع & فصل & رقم \\
\hline $\begin{array}{l}=\mathrm{IV} \\
\% \mathrm{Tl}\end{array}$ & $\begin{array}{c}r Y=\wedge \\
\%\end{array}$ & ro & $\mathrm{Va}$ & 1 \\
\hline $\begin{array}{l}=17 \\
\% 7 \varepsilon\end{array}$ & $\begin{array}{c}r 7=q \\
\%\end{array}$ & ro & $\mathrm{Vb}$ & r \\
\hline
\end{tabular}

بعد ما قام الباحث بالملاحظة

والمقابلة في المدرسـة الإبتدائية الإسلامية

الحكومية الواحدة تاناه داتار عن عملية

تعليم اللغة العربية في تلك المدرسـة،

فوجد حصول الملاحظة والمقابلة، منها

أنّ المدرّسة استعملت المصادر

التعليمية يعني كتاب المدرّس وكتاب

التلميذ، واستعملت الوسـائل التعليمية

يعني وسيلة الصورة، وأهّا رتّبت جهاز التعلّم كاملا. والمدرّسة استعملت

الطريقة السمعية الشفهية في تعليم

القراءة الجهرية يعني أن تقرأ الفقرة من

النص ثلاثة مرّات من أوّل النص إلى

آخره، ثمّ تأمر التلاميذ لتقرأ النص مرّة

واحدة جماعاة، وبعد انتهاء القراءة، تأمر

التلاميذ طلب الترجمة في النص، وبعد

ذلك تبحث المدرّسـة مع التلاميذ النص

جماعـة. وصيار حصول العملية

التعليمية في تلك الطريقة أن يكون

التلاميذ لم يستطيعوا أن يقرؤوا نص

اللغة العربية فصيحا صحيحا، فقد 
ماجستيره فهو في متغير X أنّه بحث تأثير التعلم التعاوني لأسلوب جغسئوهو، أمّا الكاتب فبحث عن تأثير الطريقة التركيبية. أما المكان البحث فاختلف بين الكاتب وبينه، أما الكاتب فبحث في المدرسة الإبتدائية الإسلامية الحكومية الواحدة تاناه داتار، وهو بحث في الماديه المدرسة العالية الإسلامية الحكومية

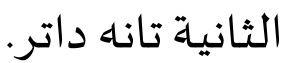

كما عرفنا أن الطريقة التركيبية قسمان هما الطريقة الحرفية والطريقة الصوتية. والطريقة الحرفية هي طريقة في تعليم القراءة للأطفال المبتدئين، تبدأ بتعليمهم أسماء الحروف الهجائية بالترتيب (ألف، باء، تاء، ثاء، ....، إلى الى الياء)، إلى أن يتمكن التلميذ من معرفة جميع حروف الهجاء بأسمائها، وينطقها نطقًا صحيحًا، ثم يكون منها كلمات

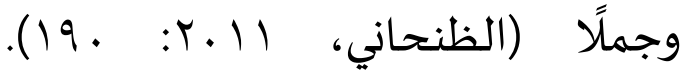
والطريقة الصوتية تبدأ هذه الطريقة بتعليم الطفل أصوات الحروف بدلا من أسمائها بحيث ينطق بحروف الكلمة أولا على انفراد مثل (ز - ر- ع)، ثم ينطق بالكلمة موصولة الحروف دفعة واحدة، وهو يتدرج في ذلك. فبعد أن يتدرب الطفل على أصهوات الحروف الهجائية
بناء على هذه المشكلة، قدّّم الكاتب علاجها باستعمال الطريقة التركيبية لترقية نتيجة تعلّمهم في دروس التمال

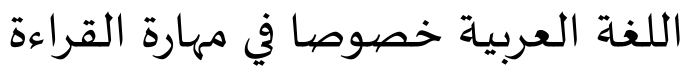
(القراءة الجهرية). الطريقة التركيبية هي التهريه التي تبدأ بالجزئيات، كالبدء بالحروف

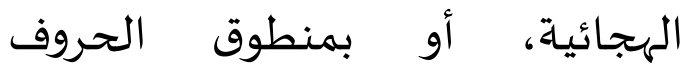

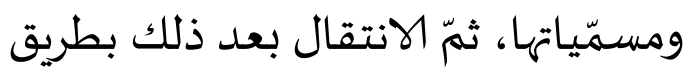
التركيب إلى المقاطع والكلمات، ثمّ بهم

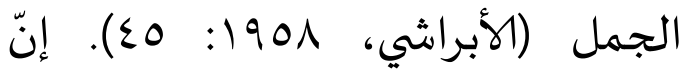
البحوث المناسبة بالمشكلات التي تتقارب ههذا البحث هي رسالة ماجستير سلمان جفري هو طالب بجامعة إمام بونجول

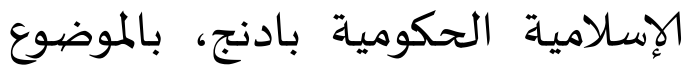

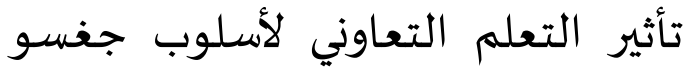
على مهارة القراءة في المدرسة العالية الإسلامية الحكومية الثانية تانه داتر.

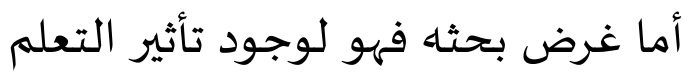

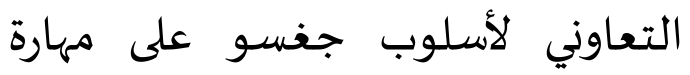
القراءة في المدرسة العالية الإسلامية الماكية الحكومية الثانية تانه داتر. أما الكاتب المباه فبحث عن تأثير الطريقة التركيبية على الى التهابه ترقية مهارة القراءة في المدرسة الإبتدائية الإسلامية الحكومية الواحدة تاناه داتار (بحث تجريبي). أما الفرق بين رسالة ماجستير الكاتب ورسالة 


$$
\text { بَ)، وذلك مع كل الحروف. }
$$

ه. يدرب المدرّس التلاميذ على قراءة وداء

الكلمة، بذكر اسم الحمرف المدرف

الأول، ثم ضبطه ونطق صوته

الناتج عن هذا الضبط، ثم تهم

ينتقل إلى الحرف الثاني ويعالجها

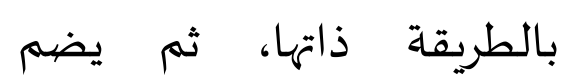

الحرفين الأول والثاني في مقطع

واحد، ثم ينطق المقطع، ثم المرين

يضم إليه الحرف الثالث، ثم

ينطق الكلمة كاملة.

ب. خطوات السير في الطريقة الصوتية:

ا. يعرض المدرّس الكلمة المراد

معرفة أصوات حروفها وبجوارها

صورة تدل عليها، مثل كلمة

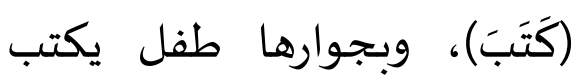

$$
\text { بالقلم. }
$$

r. يعرض المدرّس الحرف الأول

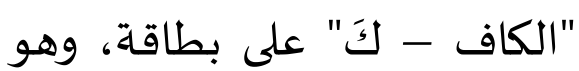

يشير إلى الصورة، وينطق (كَ،

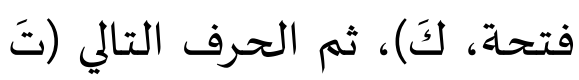

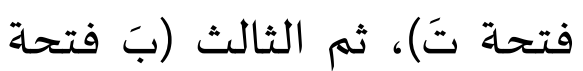

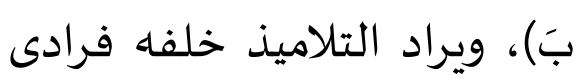

$$
\text { وجماعات. }
$$

r. يدرب المدرّس التلاميذ على ضهم

صوتين في مقطع واحد (كَتَّ..)،
ويجيد نطقها مضبوطة فتحا وضما

وكسرا، يبدأ المدرّس في تدريب الطفل

على جمع صوتين في مقطع واحد، ثم

ثلاثة أصوات ... إلخ. وهكذا حتى ينى ينتهى واحث،

إلى تأليف الكلمات من الأصوات، ثم إه

تأليف الجمل من الكلمات (مدكور،

. (1 $\varepsilon 9: Y . .7$

أما خطوات الطريقة التركيبية

فهي:

أ. خطوات السير في الطريقة الحرفية:

ا. يقوم المدرّس بكتابة الحروف

الهجائية مبتدئا بالترتيب (أ، ب، بكتون المروفي

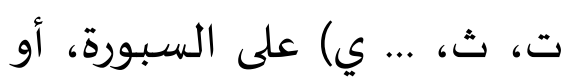

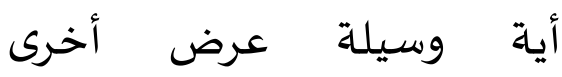

كاللوحات، أو البطاقات، أو أو أوريل

الشفافيات، أو الحاسوب.

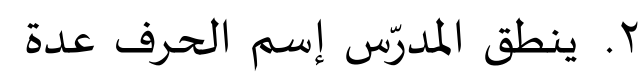

مرات، ويطالب التلاميذ بنطقه

عدة مرات، فرادى، مجموعات.

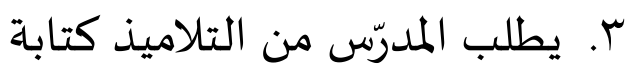

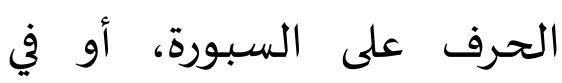

كرساتهم الخاصةة، أو سبوراتهم الحتورة

الخاصة الصغيرة.

ع. يدرب المدرّس التلاميذ على نطق احقه

الحرف مع الحركات مكسورا،

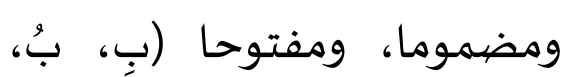


على جميع الكلمات لأنها تتألف من هذه الحروف، وفي هذا توفير للوقت

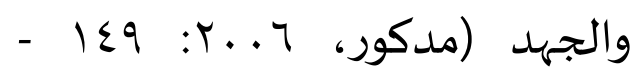
. (10.

د. يسر هذه الطريقة وبساطتها، وإذا

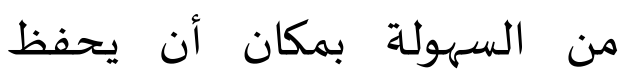

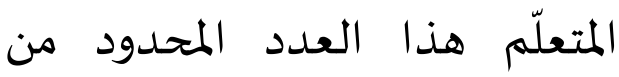
الحروف ويتعرّف على أشكالها ويربط بينها وبين أصيواتها.

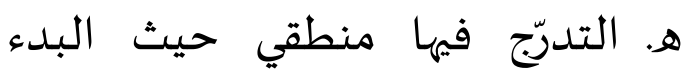
بالحروف ثمّ الكلمات فالجمل. و. تساعد المتعلّم على نطق الحروف

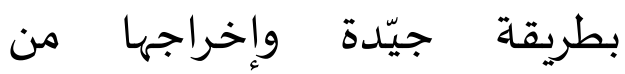
مخارجها والتمييز بينها.

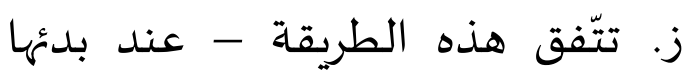

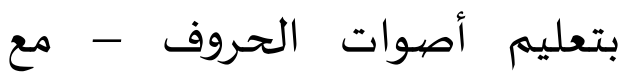

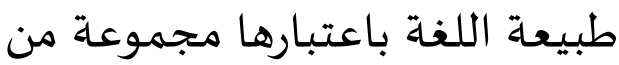

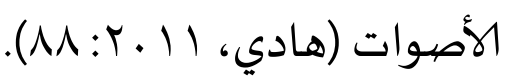
و عيوب الطريقة التركيبية هي: أ. أن أسماء الحروف الهجائية لا تعني شيئا بالنسبة للطفل، ولا ترتبط بأشياء لها معني لدياء ارتباطا طبيعيا.

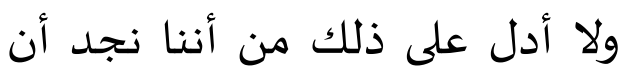
بعض الآباء حين يريدون التحدث

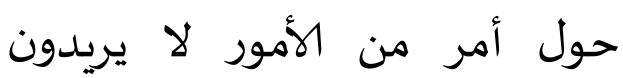
لطفلهم الصغير فهماه، يلجؤون إلى هن اهون
بحيث يبدأ بالنطق، ويكرر التلاميذ خلفها فرادى وجماعات. ع. يدرب المدرّس التلاميذ على ضم الصوت الثالث للصوتين ونطق الكلمة متصلة دون نطق الحروف

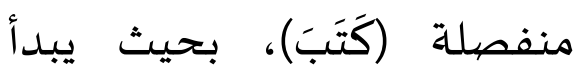

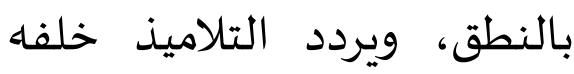
فرادى وجماعات.

ه. يقدم المدرّس للتلاميذ كلمات متشابهة في مقطع صوتى واحد

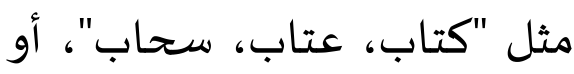

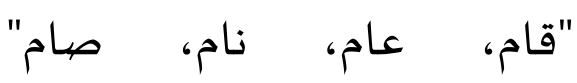

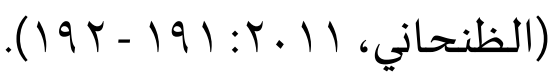
أما مزايا الطريقة التركيبية أ. أنها لا تكلف المبتدئ مشقة كبيرة

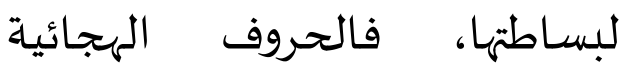
محدووة في عددها، وليس من الصعب حفظها والربط بينها وبين أصواتها. ب. أنها تتفق مع المنطق، حيث يحدث التدرج والانتقال من الجزء إلى الكل ومن الحروف إلى الكلمات، ثم إلى إلى الى الى

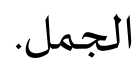
ج. أنها متى أتقن الطفل تعلم الحروف وأصواتها فإنها يستطيع أن يتعرف انه 
جر هو نفس نطق كلمة (علا) الفعل. ويلاحظ اختلاف العلاقة الدالة على الصوت من حيث الرسم وهذا يربك الطفل.

هـ إن نطق التلاميذ الذين يتعلمون بالطريقة التركيبية (هجائية كانت أو صوتياة) مفكك ومجزأ، فهم ينطقون الكلمة حرفا حرفا، وقد يتحظف

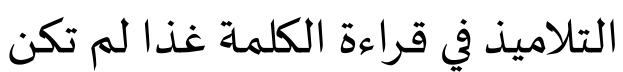
حروفها مشكولة؛ وذلك لأنهم لا لتهاء يسترشدون بمعنى الكلمة لينطقون

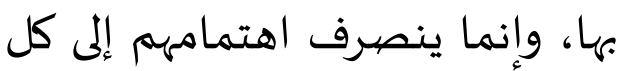
جزء من أجزاء الكلمة على حدة، وانها، كما يقرؤون الجملة كلمة كلمة. و. إن الطريقة التركيبية تركز على اكتساب المهارات الآلية كأداة لتعليم التلميذ القراءة، وليس فيها ما يرتبط بنموه ككل. وينبغي أن يرتبط النمو القرائي بنشاط التلميذ، ولعباه مع أقرائه، وما يقوم باه من رحلات، وما بلات يمر باء من خبرات شخصية، ولا يمكن أن يتحقق هذا الربط إذا كان

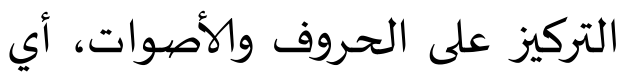

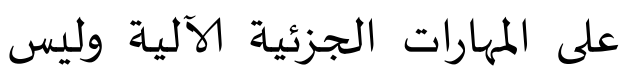

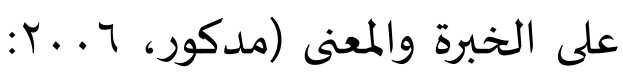
.(10) - 10 .
تهجى الكلمات دون نطقها، فالطريقة الهجائية تحول دون الطفل ومعرفة الكلمة ومعناها. ب. لا يستطيع الطفل تعلم القراءة بمجرد نطق أسماء حروف الكلمة، إذ ينبغي أن يتوصل بطريقة اسئاء حروف إلى الى

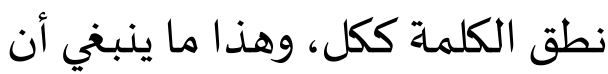
إليه الطريقة الهجائية إذا أريد للتلميذ أن يتعلم القراءة. وإذا كان الأمر كذلك فلماذا لا نبدأ بالكلمة ونؤجل تهجيها إلى مرحلة تالية ؟

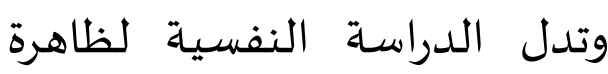
الإدراك أن الكل سابق في إدراكها على الجزء، بل وأن الجزء يتحدد الجدران إن إدراه معناه من الكل الذي ينتهى إليه.

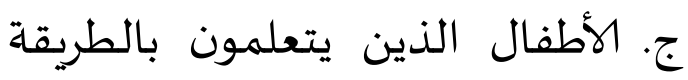

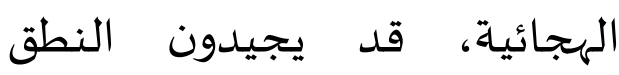
بالكلمات لأنهم تعودوا أن يفحصوا لهونائه

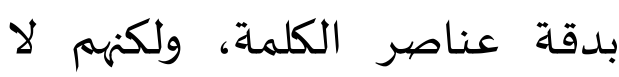
يهتمون بمعنى المقروء اهتماما كافيا،

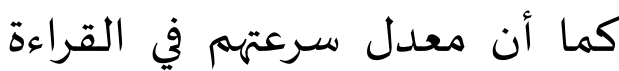
منخفض جدا. د. تعاني الطريقة الصوتية من وجود الكلمات تشتمل على صوت الحرف المتحرك ولكن رسمها مختلف، مثال ذلك أن نطق كلمة (على) وهي حرف 
الحروف من مخارجها الصحيحة، وهي أحسن وسيلة لإتقان النطق وإجادة الأداء وتمثيل المعنى. وقد كانت فيما مضى موضع العناية في المدارس، ولكنّ

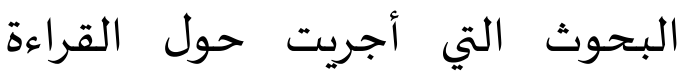

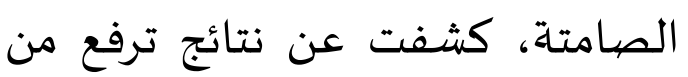

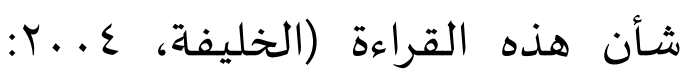

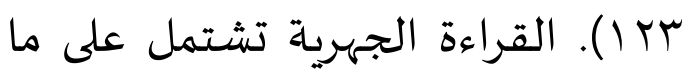

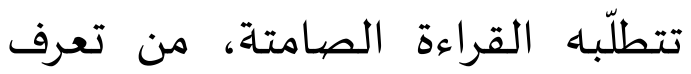
بصري للرموز الكتابية، وإدراك عقلي

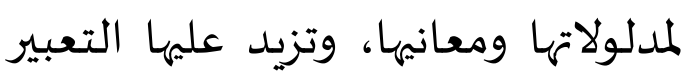
الشفهي عن هذه المدلولات والمعاني، بنطق الكلمات والجهر بها، وبذلك كانت

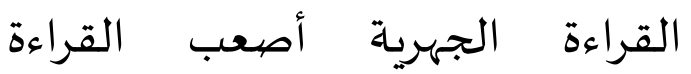

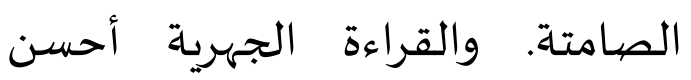
وسيلة لإتقان النطق، وإجادة الأداء، وتمثيل المعنى، ولاسيما في الصفوف ولكان

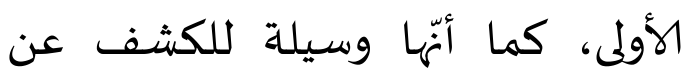
أخطاء التلاميذ في النطق، فيتسنى علاجها. وهي أيضا تساعد في الصفوف العليا على تذوّق الأدب بتعرّف نواحي الانسجام الصيوتي والموسيقا اللفظية. وهي وسيلة لتشجيع التلاميذ ذوي الخوف والتهيب، وعلاج هذه الداء فيهم،

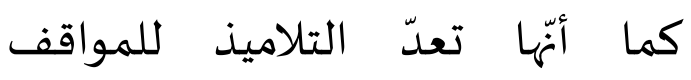

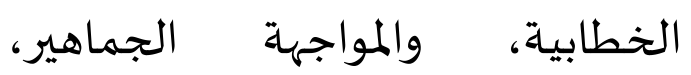

القراءة هي عملية التعرّف على الرموز المطبوعاة، ونقطها نطقا صحيحا

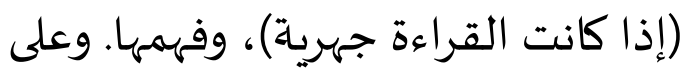
هذا فهي تشمل التعرّف، وهو الاستجابة البصرية لما هو مكتوب، والنطق، وهو تحويل الرموز المطبوعة التي تمّت رؤيتها إلى أصوات ذات معنى، والفهم، أي الي ترجمة الرموز المدركة وإعطائها المعاني. القراءة بهذا المفهوم عملية معقّدة، ففيها تعرّف، وففيها نطق، ففيها فهم. وبالرغم أهمّية التعرّف في القراءة إلاّ أنّه وسيلة ليس غاية في ذاته، فالتعرّف

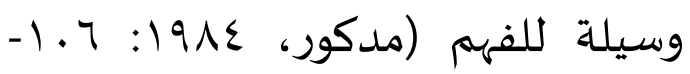

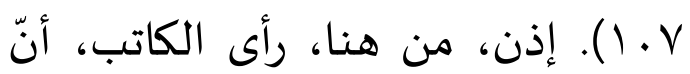

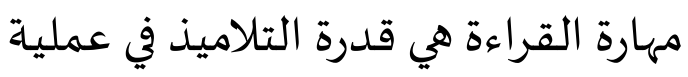
التعرّف على الرموز المطبوعاة، ونقطها

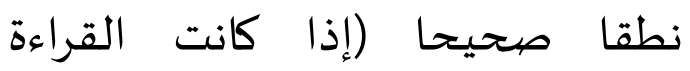
جهرية)، وفهمها. فهي تشمل التعرّف،

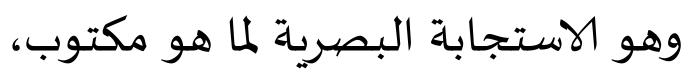
والنطق، وهو تحويل الرموز المطبوعة

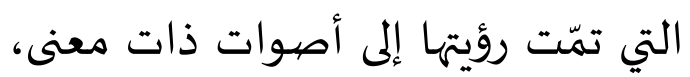
والفهم، أي ترجمة الرموز المدركة وإعطائها المعاني. القراءة الجهرية هي القراءة بصوت مسموع، ونطق واضح صحيح لإكساب الطفل صعب النطق وإخراج 
لا معنى لها بذاتها فإنّ هذه الطريقة لا تركز في البدء على المعنى (مدكور، بله

(1) (1)

\section{طريقة البحث}

أمّا نوع البحث الذي استعمله

الكاتب فهو بحث تجريبي، واستعمل منهج شباه تجريبي مع تصميم مجموعاة

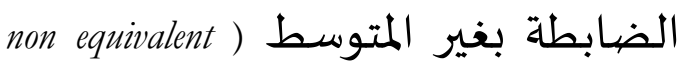
control group design بغير Random والطريقة المستعملة هي طريقة إحصائية لأنّاء بحث عن تأثير

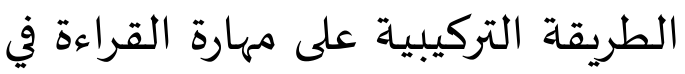
المدرسة الإبتدائية الإسلامية الحكومية الواحدة تاناه داتار (بحث تجريبي). أما مجتمع البحث في هذا البحث فهو كل التلاميذ في المدرسـة الإبتدائية الإسلامية الحكومية الواحدة تاناه داتار، يعني \&OV البحث فهي التلاميذ في الفصل

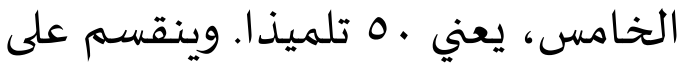

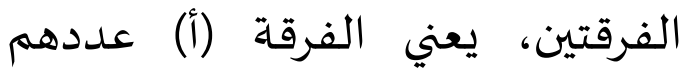
خمسة وعشرون (Yo) ويسمّى الفرقة الفرية الضيابطة، والفرفة (ب) عددهم خمسـة وعشرون (ro) تلميذا ويسمّى الفرقة التجريبية. وأخذ الكاتب عينة تجريبية باستعمال العشوائية التطبيقية
والحديث إلى الجماعة (الركابي، 1997: ( ) إنّ الغرض من القراءة الجهرية أن يمرن المدرّس التلاميذ على القراءة إند العراءه الجهريه بصوت يسمع حتّى يستطيع أن يصلح

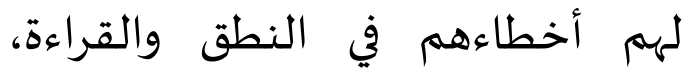

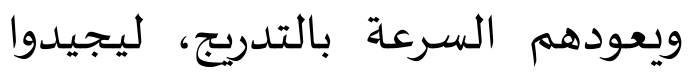

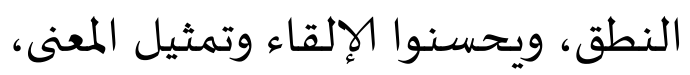

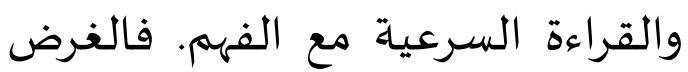
من القراءة الجهرية تعود التلاميذ حسن فئن

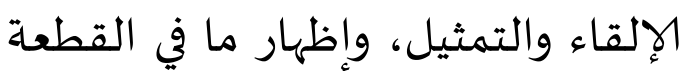
من جمال حتّى يجد المستمعون لذة في

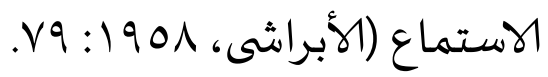

أن الطريقة التركيبية هي التي تبدأ بالجزئيات، كالبدء بالحروف البهديه

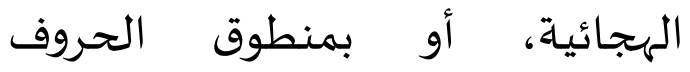

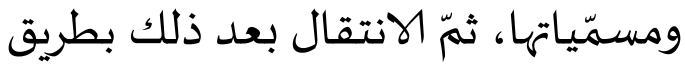
التركيب إلى المقاطع والكلمات، ثمّ بهم

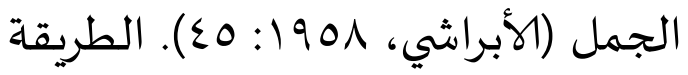
التركيبية هي تبدأ بتعليم الجزئيات، كالبدء بتعليم الحروف الأبجدية تبدية

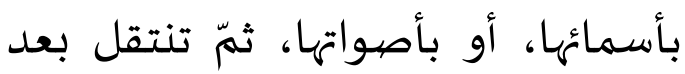

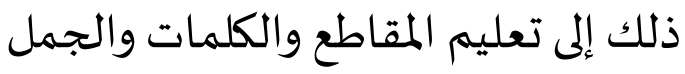

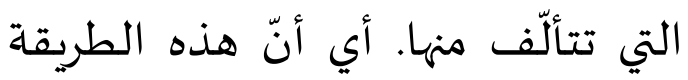
تبدأ من أصغر وحدات ممكنة وتنتقل إلى الوحدات الأكبر. ولما كانت الجزئيات 
عينة البحث، فاستعمل بالحاسوب باسم SPS 25 . تحليل البيانات هو باتهيك سعي الكاتب للحساب والبحث عن

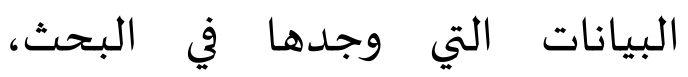
واستطاع أن يستخدم تحليلا مناسبا

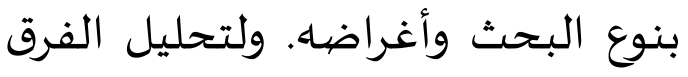
بين نتيجة تعليم مهارة القراءة باستعمال الطريقة التركيبية ونتيجة تعليم مهارة القراءة بغير استعمال

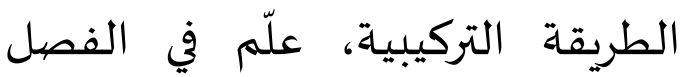
الخامس (أ) بطريقة السمعية الشفهية فقام امتحان القراءة (القراءة الجهرية)

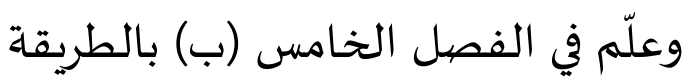
التركيبية، فامتحنهم بالقراءة (القراءة

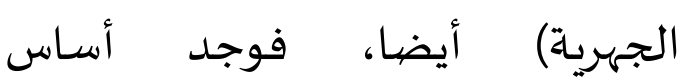
النتيجتين، هما نتيجة القراءة بالطريقة التركيبية (X) ونتيجة القراءة بطريقة السمعية الشفهية (Y). ولتحليل النتيجتين استعملهما الرمز لمعرفة الميه

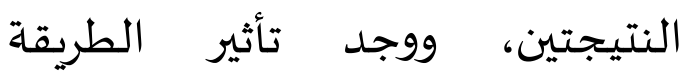
التركيبية على مهارة القراءة في المدرسة

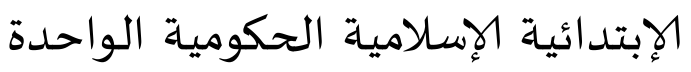

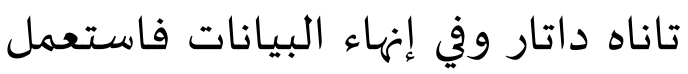

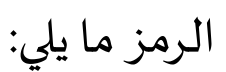$$
\mathrm{t}_{\mathrm{o}}=\underline{\mathrm{M}_{\mathrm{D}}}
$$$$
\mathrm{SE}_{\mathrm{MD}}
$$

وأمّا خطوات إحصائيتها فهي ما
}

.(Random Sampling)

في هذا البحث استعمل الكاتب

تحليل "Tes " to أمّا الخطوات في البحث

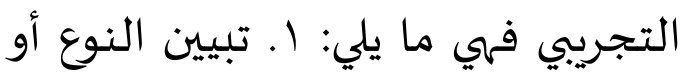
الشكل، r. تعيين الفرقة المبحوثة، r. آلة البحث، ع. التجريبة، 0. لشرط التحليل. قبل أن يستعمل الكاتب امتحان الفرضاة يحتاج إلى فحص البيانات لمتغيرات X و Y. في هذا الفحص عمل امتحان نورمالتس (Uji Normalitas) وامتحان هموجنيهس (Uji Homogenitas). أمّا الغرض من امتحان هموجنيهن الخانين فهو لتأخذ الخلاصة بصحيحة وتمامة. واستعمل امتحان نورمالتس بامتحان ليليفورس وامتحان هموجنيتس بامتحان (Liliefors) بارتليت (Bartlett). يستعمل امتحان نورمالتس البيانات بكيفية امتحان ليلفور (Liliefors) وامتحان Chi-Squere. لمعرفة نورمال أو غير نورمال من ونحان الامتحان استعمل في إعطائه إلى عينة البحث، فحلل بالحاسوب باسم 25. و وامتحان هموجنيتس على فرقة باستعمال طريقة بارتليت (Bartlet). لمعرفة هموجنيتس أو غير هموجنيتس بارس من الامتحان استعمل في إعطائه إلى همئل 
التركيبية على ترقية مهارة القراءة في

الفرقة التجريبية (Eksperimental)، فقام بالامتحان على التلاميذ ووجد أقصى النتائج بو وأدلى النتائج Vo. فوجد نتائجهم كما يلي:

\begin{tabular}{|c|c|c|}
\hline \\
\hline متوسطة & عدد نتائج الطريقة & عدد \\
\hline النتيجة & المزدوجة (XX) & التلاميذ \\
\hline$r \varepsilon, \Lambda T$ & r. .1 & ro \\
\hline
\end{tabular}

وهذه نتائج ترقية مهارة

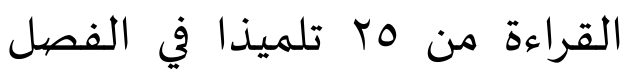
الخامس، يعني نتائج ترقياة مهارة القراءة بالطريقة التركيبية في الفرقة التجريبية. ومن الجدول السابق، وضح لنا أنّ التلميذ الذي حصل التيل على أقصى النتائج تلميذ واحد يعني

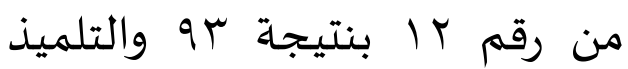
الذي حصل على أدنى النتائج

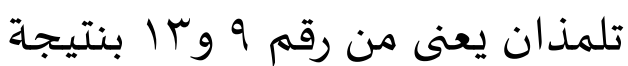
Vo. ونظر الكاتب نتيجة ترقية مهارة

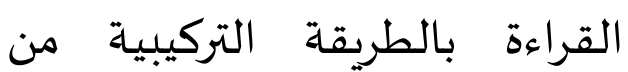
الحاسوب فيما يلي:

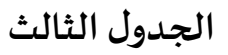

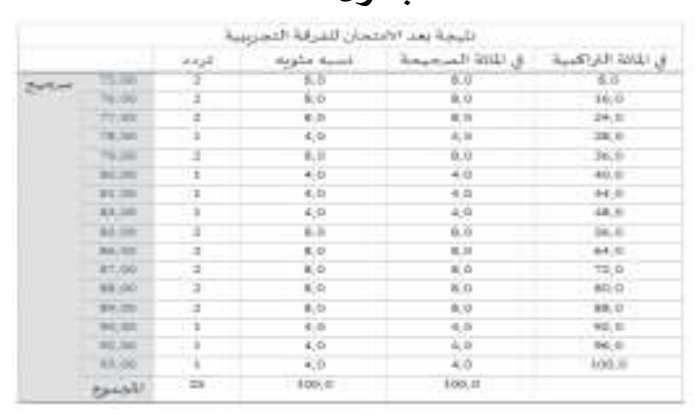

أ. بحث الكاتب عن تفريق بين

النتيجتين باستعمال الرمز D=X-Y ب. بحث الكاتب عن نتيجة D حتيّى يحصل على ئ بحلى ج. بحث الكاتب عن معدل التفريق

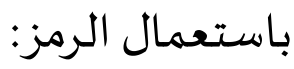
$\mathrm{M}_{\mathrm{D}}=\sum \underline{\mathrm{D}}$ $\mathrm{N}$

د. ضياعف الكاتب نتيجة التفريق D ثمّ يبلنها حتّى يحصل نتيجة صD

Defiasi (SD Standar dari Diference

$$
\mathrm{SD}_{\mathrm{D}}=\sqrt{\mathrm{N}} \underline{\mathrm{D}}^{2}-\left(\sum_{\mathrm{N}} \mathrm{D}^{2}\right)
$$

Standar eror dari زحث الكاتب عن باستعمال mean of diference ( $\left.\mathrm{SE}_{\mathrm{MD}}\right)$

$$
\mathrm{SE}_{\mathrm{MD}}=\frac{\mathrm{SD}_{\mathrm{D}}}{\sqrt{\mathrm{N}-1}} \quad \text { الرمز }
$$

ح. بحث الكاتب عن to باستعمال الرمز : $\mathrm{t}_{\mathrm{o}}=\underline{\mathrm{M}}$

ط. إعطاء التفسير لنتيجة (Sudijono) to .$(r \cdot V: Y . .0$

\section{نتيجة البحث ومناقشتها صفة البيانات}

أ. صفة الطريقة التركيبية على ترقية

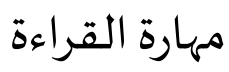
جرب الكاتب الطريقة 
النتائج م م وأدنى النتائج • 7. فوجد نتائجهه كما في الجدول التالي:

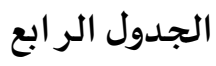

\begin{tabular}{|c|c|c|}
\hline متوسطة & عدد نتائج طريقة & 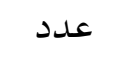 \\
\hline النتيجة & السمعية الشفهية (Y) & التلاميذ \\
\hline$V T, \Lambda \Lambda$ & MKT & ro \\
\hline
\end{tabular}

وهذه نتائج ترقية مهارة

القراءة من 0 م تلميذا في الفصل الخامس، يعني نتائج ترقية مهارة القراءة بطريقة السمعياة الشفهية في الفرقة الضيابطة. ومن الجدول السابق، وضح لنا أنّ التلميذ الذي حصل على أقصى النتائج تلميذ واحد يعني من رقم 10 بنتيجاة والتلميذ الذي حصل على أدنى النتائج تلميذ واحد أيضا يعنى من رقم ب ب بنتيجة . 7. وهكذا نتيجة مهارة القراءة بطريقة السمعية الشـهية من الحاسـوب فيما يلي :

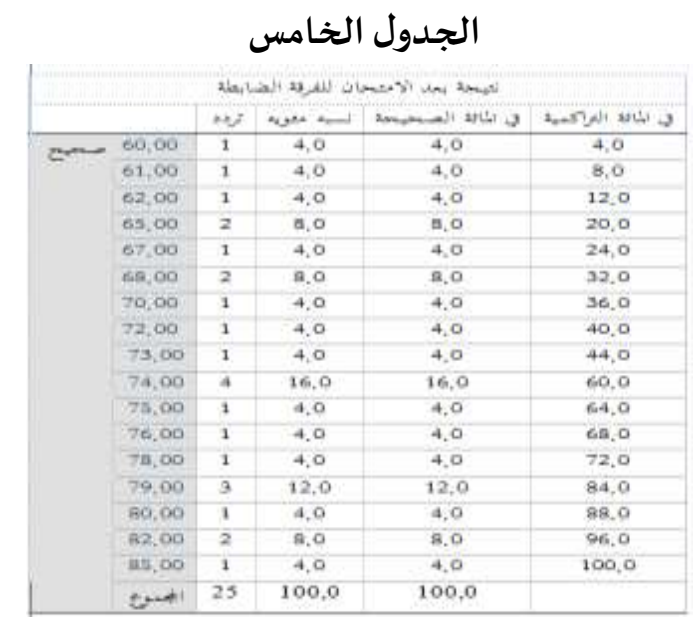

وهكذا نتيججة ترقية مهارة القراءة بالطريقة التركيبية. وبين الجدول السابق عن نتائج ترقية مهارة القراءة من Oم تلميذا بعدما حلل الكاتب بالحاسوب. ووجد فيـ أقصى النتائج وأدناها. فأما أقصى النتائج فحصل على تلميذ واحد

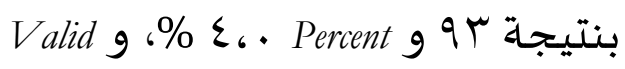
Cumulative Percent g 9 \&.. Percent

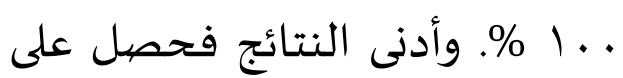

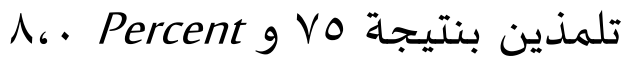
9 6 N.. Valid Percentg .\% \% ^ ، . Cumulative Percent ب. صفة طريقة السمعية الشفهية الطريقة السمعية الشفهية هي الطريقة استعملت المدرّسـة في المدرسة الإبتدائية إسبلامية الحكومية الواحدة تاناه داتار. عادة

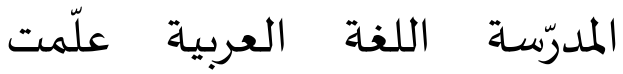
التلاميذ كمثل هذه الطريقة فى مهارة القراءة. استعمل الكاتب الامتحان في الفرقة الضـابطة لينظر نتيـاة ترقية مهارة القراءة بطريقة السمعية الشفهية. من بيان نتيجة ترقية مهارة القراءة بالطريقة

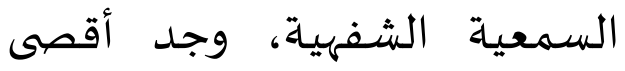


0,7^ᄉrq = (SD)

المطلق (Ekstreme Absolut Most)

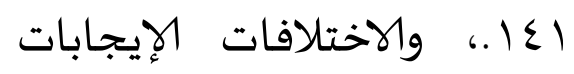

. ITY = (Differences Positif)

والاختلافات

‘-.|乏।= (Differences Negatif)

Test ) واختبار إإحصيائية

(Statistic $.200=$ Asymp. Sig. (2-tailed)

وهكذا حاصل الامتحان نورمالي في متغير X Xتيججة مهارة القراءة بالطريقة التركيبية). وما أصبح ذلك، لمعرفة نورمالي أو غير نورمالي من الامتحان استعمل في إعطاء الامتحان إلى العينة البحث، حلل بالحاسوب باسم إعم إلماء SPSS 25 معنى وانحراف المعياري (SD) r ا . 7,97، وأكثر تطرف المطلق .. $Y \mathcal{E}=($ Ekstreme Absolut Most $)$ والاختلافات ... $\vee \wedge=($ Differences Positif $)$ والاختلافات . -.1 I $\varepsilon=$ (Differences Negatif) Test ) واختبار إلحصيائية
وهكذا نتيججة ترقية مهارة القراءة بالطريقة السمعية الشفهية. وبين الجدول السـابق عن نتائج ترقية مهارة القراءة من 0ب تلميذا بعدما حلل الكاتب بالحاسوب ووجد فيه أقصى النتائج وأدناها. فأما أقصى النتائج فحصل على تلميذ

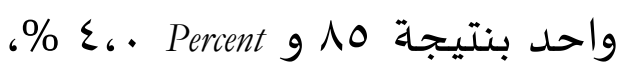
Cumulative g \& \&.. Valid Percentg Percent فحصل على تلميذ واحد أيضا

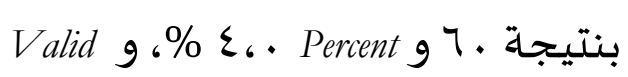
Cumulative Percent g 9 \&。. Percent $\% \varepsilon_{6}$. ج. امتحان لشرط التحليل

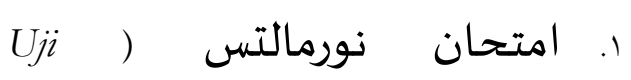
(Normalitas

استعمل الكاتب امتحان

نورمالي البيانات بكيفية امتحان ليليفورس (Liliefors) وامتحان خي مربع (Chi-Squere). لمعرفة

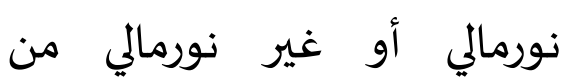
الامتحان استعمل في إعطائه إلى عينة البحث، فحلل بالحاسـوب باسم 25 SPSS فوجد عن حاصلته هي معنى (Mean) ع . . . . . . وانحراف المعياري 
مربع (Chi-Square Test). فظهر لنا حاصل امتحان هموجني كما في الجدول الأتي:

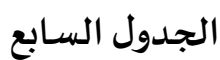

\begin{tabular}{|c|c|c|c|}
\hline \multirow[t]{2}{*}{$x, x \rightarrow-1$} & \multicolumn{2}{|c|}{ 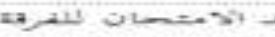 } & $\mathrm{x}+\mathrm{n}=$ \\
\hline & NI & $\frac{N}{N}$ & - \\
\hline 75.00 & 2 & 1.6 & .4 \\
\hline 76.90 & 2 & 1,6 & -4 \\
\hline 75,00 & 2 & 1,6 & .4 \\
\hline 78.90 & 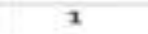 & 1,6 & -.0 \\
\hline $29.0 \mathrm{co}$ & 2 & 1.6 & .4 \\
\hline$B D, 00$ & 3 & 1.6 & -6 \\
\hline $81, \mathrm{crc}$ & 1 & 1,6 &,- 0 \\
\hline 83.00 & I & 1. 6 & -.6 \\
\hline $0=0,00$ & $z$ & 1.0 & .4 \\
\hline 80.00 & 2 & 1.6 & .4 \\
\hline 85.00 & $z$ & 1,6 & -4 \\
\hline 88,00 & 2 & 1,6 & .4 \\
\hline 199.00 & 2 & 1,6 & .4 \\
\hline 90.00 & 1 & 1,6 & -6 \\
\hline 22,00 & 1 & 1,6 & -.6 \\
\hline 93.00 & 1 & 1.6 & -.0 \\
\hline$=0 .+1$ & 25 & & \\
\hline
\end{tabular}

وما أصبح ذلك، في متغير

قام الكاتب امتحان

هموجنيتس بامتحان Chi-Square

$$
\text { Test }
$$

$$
\text { الجدول الثامن }
$$

\begin{tabular}{|c|c|c|c|}
\hline & ملاحظة N & Nت متوقع N & متبقي \\
\hline 60,00 & 1 & 1,5 &,- 5 \\
\hline 61,00 & 1 & 1,5 &,- 5 \\
\hline 62,00 & 1 & 1,5 &,- 5 \\
\hline 65,00 & 2 & 1,5 &, 5 \\
\hline 67,00 & 1 & 1,5 &,- 5 \\
\hline 68,00 & 2 & 1,5 &, 5 \\
\hline 70,00 & 1 & 1,5 &,- 5 \\
\hline 72,00 & 1 & 1,5 &,- 5 \\
\hline 73,00 & 1 & 1,5 &,- 5 \\
\hline 74,00 & 4 & 1,5 & 2,5 \\
\hline 75,00 & 1 & 1,5 &,- 5 \\
\hline 76,00 & 1 & 1,5 &,- 5 \\
\hline 78,00 & 1 & 1,5 &,- 5 \\
\hline
\end{tabular}

نتيجة بعد الامتحان للفرقة الضابطة
(Statistic $.200=$ Asymp. Sig. (2-tailed) وهكذا حاصل الامتحان نورمالي في متغير X فظهر لنا حاصل في

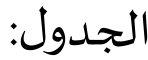

\begin{tabular}{|c|c|}
\hline \multicolumn{2}{|c|}{ اختبار عينة واحدة قلموغوروف سميرنوف } \\
\hline & تتيجة بعد الومتحان للفرقة \\
\hline $\mathrm{N}$ & 25 \\
\hline معنى المدرسات & 83,2400 \\
\hline a,b الحراف المعياري الكادية & 5,68829 \\
\hline مطلق معظم & .141 \\
\hline إيكاني الديخلالفات & .132 \\
\hline سليي لقموى & -.141 \\
\hline الختيار الإمصائية & .141 \\
\hline أهمية الذيل & $.200^{\varepsilon, d}$ \\
\hline
\end{tabular}

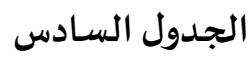

من الجدول السـابق ظهر

لنا أن الفرقة في هذه البحث أصلاء من العينة نورمال، لذلك شرط نورمال مقبول.

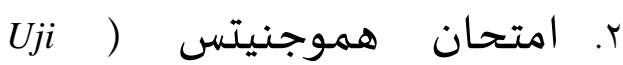

\section{(Homogenitas}

امتحان هموجني على

فرقة باستعمال الطريقة بارتليت (Bartlet) هموجني من الامتحان استعمل الكاتب في إعطائه إلى العينة البحث، فاستعمل بالحاسـوب باسم SPSS 25 فوجد عن متغير من التحليل بالامتحان خي X 
الامتحان على الفرقتين. ثم يقارن

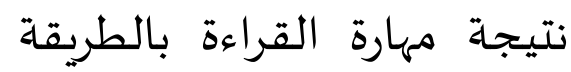

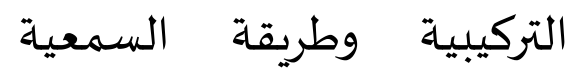
الشفهية. بعدما قام الامتحان فحصل على نتيجة مهارة القراءة

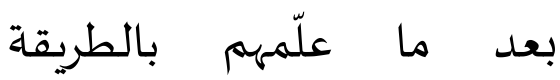
التركيبية. وبعد ذلك جمع في

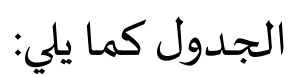

\begin{tabular}{|c|c|c|c|c|c|}
\hline $\mathrm{D}^{2}$ & $\mathrm{D}=(\mathrm{x}-\mathrm{y})$ & $Y$ & $\mathrm{X}$ & الثاديذ & ترت \\
\hline $3 \times$ & 3 & 65 & 76 & 1 & 1 \\
\hline$t$ & $r$ & 78 & 80 & $r$ & $r$ \\
\hline 29 & $r$ & 79 & 86 & $r$ & $r$ \\
\hline 77 & 7 & 82 & 88 & $\varepsilon$ & $\varepsilon$ \\
\hline$t$ & $r$ & 75 & 77 & 0 & $\circ$ \\
\hline$Y=$ & $=$ & 85 & 90 & 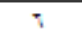 & 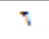 \\
\hline $7 t$ & A & 70 & 78 & $\mathrm{v}$ & $v$ \\
\hline 3 & 3 & 67 & 77 & A & 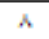 \\
\hline \pm 9 & $\mathrm{Y}$ & 68 & 75 & 9 & 9 \\
\hline 3 & 7 & 80 & 81 & 1. & 1. \\
\hline$\pi$ & 7 & 79 & 85 & 11 & 11 \\
\hline$\gamma .9 t$ & $T r$ & 61 & 93 & ir & ir \\
\hline$\cdots$ & 3 & 65 & 75 & ir & ir \\
\hline$r=$ & $=$ & 74 & 79 & $1 \leq$ & 18 \\
\hline$Y=$ & $=$ & 82 & 87 & 10 & 10 \\
\hline 179 & 15 & 76 & 89 & 17 & 17 \\
\hline 197 & $1 t$ & 74 & 88 & iv & iv \\
\hline 179 & 15 & 72 & 85 & $1 \mathrm{~A}$ & is \\
\hline$\cdots$ & 3 & 79 & 89 & 19 & 19 \\
\hline$r$ & 19 & 73 & 92 & $r$. & $r$. \\
\hline rA9 & IV & 62 & 79 & Y & Yi \\
\hline$y=7$ & 17 & 60 & 76 & $r y$ & $r Y$ \\
\hline$r Y t$ & iA & 68 & 86 & $r r$ & $r Y$ \\
\hline AI & 9 & 74 & 83 & $r t$ & $r t$ \\
\hline 179 & 17 & 74 & 87 & $r=$ & $Y=$ \\
\hline $\begin{array}{l}\sum D^{2} \\
= \\
\text { TYA }\end{array}$ & $\sum \mathrm{D}=\mathrm{r} a$ & $\begin{array}{l}Y= \\
\text { WYY }\end{array}$ & $\mathrm{X}=2081$ & & \\
\hline
\end{tabular}

وفي انتهاء السابقات استعمل الكاتب الرمز T test

\begin{tabular}{c|c|c|c}
\hline 79,00 & 3 & 1,5 & 1,5 \\
\hline 80,00 & 1 & 1,5 &,- 5 \\
\hline 82,00 & 2 & 1,5 &, 5 \\
\hline 85,00 & 1 & 1,5 &,- 5 \\
\hline ع المجمو & 25 & & \\
\hline
\end{tabular}

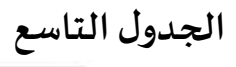

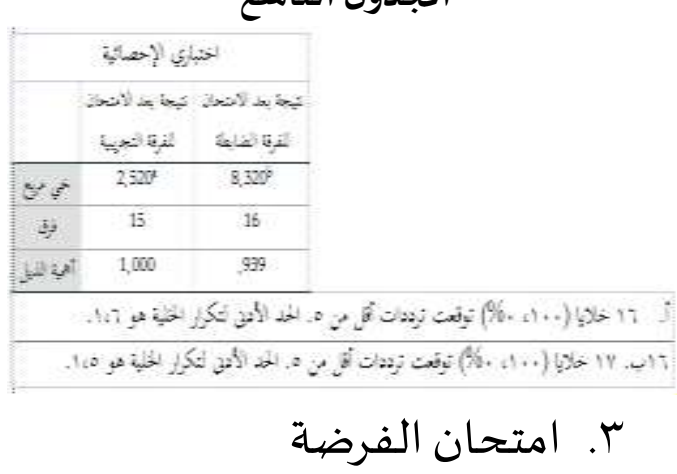

الفرض العمليـة التي

جاءت من المشكلات القديمة فهي فيما يأتي:

أ) وجد أيجير الطريقة التركيبية في ترقية

$$
\text { مهارة القراءة (H1). }
$$

ب) وجد تأثير سلبيّ (-) الطريقة السمعياة الشفهية في ترقية

$$
\text { مهارة القراءة (H2). }
$$

في إجذهراءات

استعمل الكاتب التجربية بإجراء البحث التجربي في الفرقة الضيابطة فيه مهارة القراءة بطريقة السمعية الشفهية وفي الفرقة التجريبية بالطريقة التركيبية. بعد ذلك استعمل بلك 
و) بحث الكاتب عن Standar eror

dari mean of diference $\left(\mathrm{SE}_{\mathrm{MD}}\right)$

$$
\text { باستعمال الرمز : }
$$$$
\mathrm{SE}_{\mathrm{MD}}=\frac{\mathrm{SD}_{\mathrm{D}}}{\sqrt{ } \mathrm{N}-1}
$$$$
=\underline{7, \Lambda\urcorner}
$$

$\sqrt{\text { ro- }}$

$$
=\frac{7,17}{\sqrt{r \varepsilon}}
$$$$
=\underline{7,17}
$$$$
\varepsilon, 19
$$$$
=1, \varepsilon \text {. }
$$

ز) بحث الكاتب عن to باستعمال

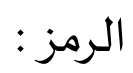

$$
\mathrm{t}_{\mathrm{o}}=\frac{\mathrm{M}_{\mathrm{D}}}{\mathrm{S}}
$$$$
=1 \text {. }
$$

$1, \varepsilon$.

$$
=V, 1 \varepsilon
$$

أما تنيـجة تحليل من

$$
\text { الحاسوب فيما يلي: }
$$$$
\text { الجدول الحادى عشر }
$$

جدول إحصاءت عينة واحدة (امتحان t)

$$
\text { بالحاسوب }
$$

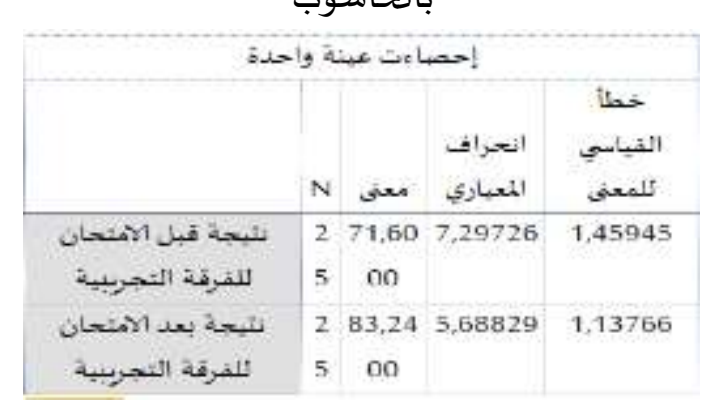

$$
\mathrm{t}_{\mathrm{o}}=\frac{\mathrm{M}_{\mathrm{D}}}{\mathrm{SE}}
$$

وأما خطوات إحصيائيتها

فهي ما يلي:

أ) بحث الكاتب عن تفريق بين

النتيجتين باستعمال الرمز

$$
\mathrm{D}=\mathrm{X}-\mathrm{Y}
$$

ب) بحث الكاتب عن نتيجة D

حتى يـحصل على

ج) بحث الكاتب عن معدل

التفريق باستعمال الرمز :

$$
\mathrm{M}_{\mathrm{D}}=\frac{\sum \mathrm{D}}{\mathrm{N}}
$$

$$
=\text { ro. }
$$

ro

$$
=1 \text {. }
$$

د) ضياعف الكاتب نتيججة

التفريق D ثم بلغها حتى

$$
\text { يحصل نتيجة }
$$

ه) بحث الكاتب عن (SDD)

Defiasi Standar dari Diference

$$
\text { باستعمال الرمز : }
$$

$$
\begin{aligned}
& \mathrm{SD}_{\mathrm{D}}=\sqrt{ } \sum \mathrm{D}^{2}-\left(\sum \underline{\mathrm{D}}\right)^{r} \\
& =\sqrt{\text { ryVA- }(\text { ro. })^{r}} \\
& \text { ro ro } \\
& =\sqrt{1 \varepsilon V, 1 Y-1 \ldots} \\
& =\sqrt{\varepsilon V, 1 T} \\
& =7, \wedge 7
\end{aligned}
$$


فالفرضية العدمية مردودة والفرضة التبادلية مقبولة، المراد بها أن هناك اختلاف بين نتيجة الامتحان الأول (نتيجة مهارة القراءة بطريقة السمعية

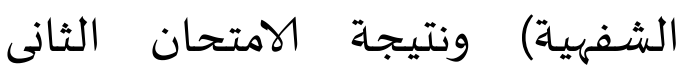

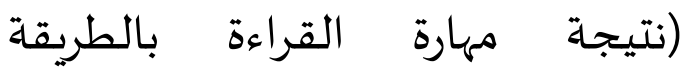
التركيبية).

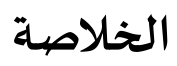

من نتيجة البحث السابق،

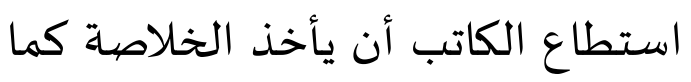

أ. إنّ الطريقة التركيبية تستطيع أن تؤثر على مهارة القراءة تأثيرا إيجابيّا في المدرسة الإبتدائية الإسلامية الحكومية الواحدة تاناه داتار. ب. هناك الفرق بين نتيجة التلاميذ التي علّمت باستعمال الطريقة التركيبية ونتيحة التلاميذ التي علّمت بطريقة السمعية الشفهية في ترقية مهارة القراءة في المدرسة الإبتدائية الإسلامية الحكومية الواحدة تاناه داتار، أما الفرق هنا فهو أن نتيـجة الإسلاه التلاميذ في ترقية مهارة القراءة باستعمال الطريقة التركيبية أعلى من نتيجة التلاميذ باستعمال طريقة

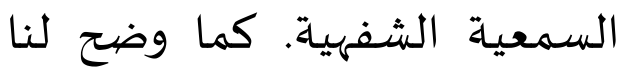

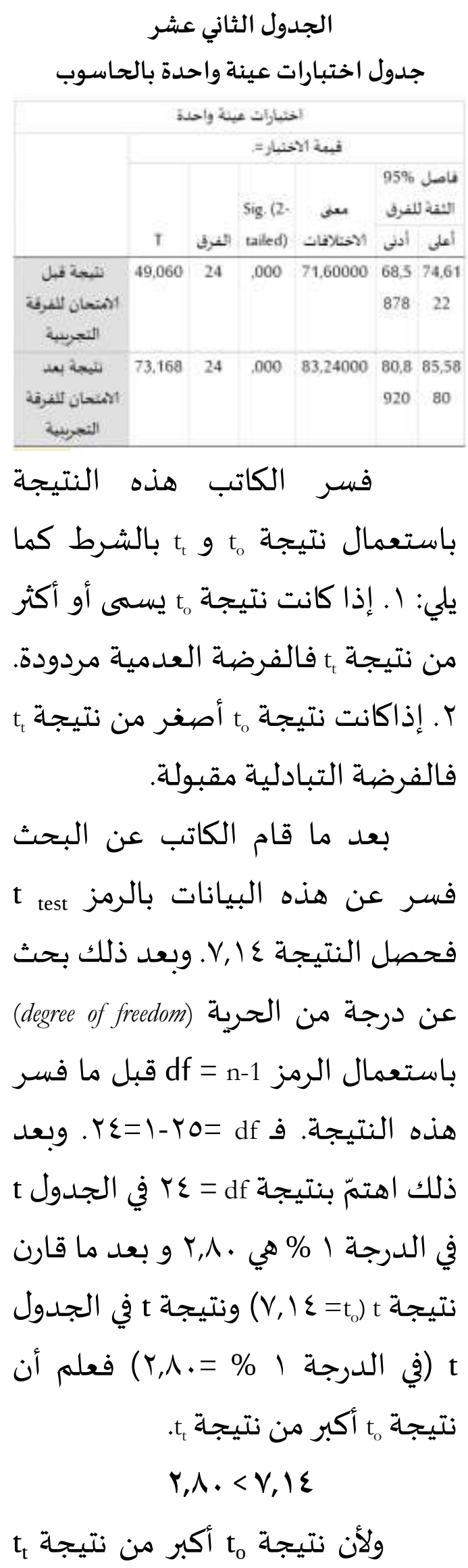

باستعمال نتيجة t g g t t بالشرط كما يلي: 1. إذا كانت نتيجة to يسمى أو أكثر من نتيجة t فالفرضية العدمية مردودة.

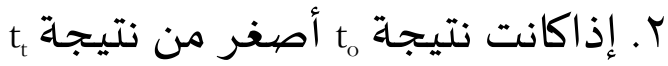
فالفرضة التبادلية مقبولة. بعد ما قام الكاتب عن البحث test فسر عن هذه البيانات بالرمز فحصل النتيجة ع ا V. وبعد ذلك بحث عن درجة من الحرية (degree of freedom) باستعمال الرمز df = n-1 قبل ما فسر هذه النتيجة. ف d

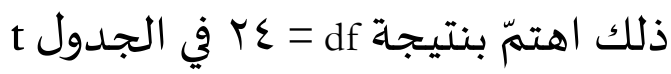

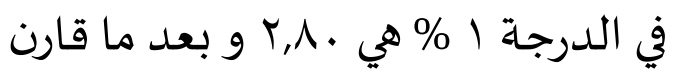

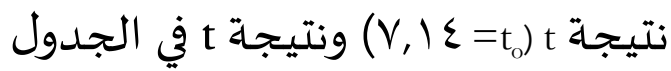

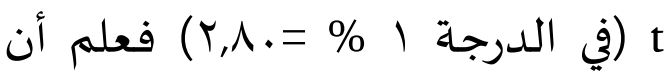

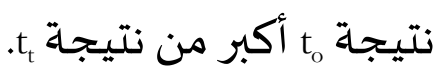
$r, \Lambda .<V, 1 \varepsilon$ tht ألأن نتيجة to 
مكتبة الفلاح.

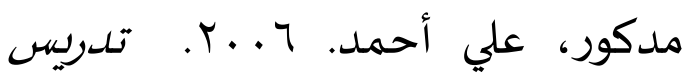

$$
\begin{aligned}
& \text { فنون اللغة العببية، القاهر: دار } \\
& \text { الشواف. }
\end{aligned}
$$

هادي، نور، البا. الموجه لتعليم المهارات اللنوية لغير الناطقين

مها. مالانق: جامعة مولانا مالك الك

$$
\text { إبراهيم الإسلامية الحكومية. }
$$

فيما يلي : (أ) وجد أقصى النتائج في ترقية مهارة القراءة بالطريقة

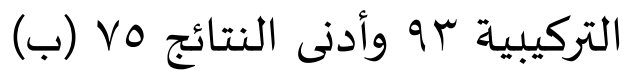
وجد أقصى النتائج فى مهارة القراءة بطريقة السمعية الشفهية مر وأدنى النتائج .7.

الأبراشى، محمد عبد. 1901. الطرق الخاصة في التوبية لتدريس اللغة العربية والدين. القاهرة: مكتبة الأنجلو المصرية.

$$
\begin{aligned}
& \text { الخليفة، حسن جعفر . ع. . ז. فصول } \\
& \text { في تلدرليس اللغة العربية: ابتدائي } \\
& \text { - متوسط - ثانوي. الرياض: }
\end{aligned}
$$

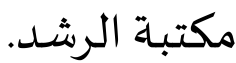

الركابي، جودت. 1997 ـ طرق تدريس

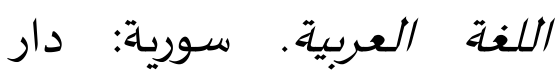

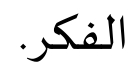

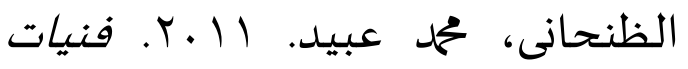

$$
\begin{aligned}
& \text { تعليم القراءة في ضيوء الأدوار } \\
& \text { الجبديدة للمعلمر والمتعلمه، } \\
& \text { القاهرة: عالم الكتب. } \\
& \text { مدكور، علي أحمد. ع1911. تلدريس } \\
& \text { فنون اللغة العببية. الكويت: }
\end{aligned}
$$

